\title{
Parálisis Cerebral Infantil, su presentación clínica en pacientes atendidos en el CRIT Aguascalientes en el periodo del 2003 al 2013
}

\author{
del Valle-Morales Aída *, Espinosa-Barragán Olivia *, Mendoza Alejandro Gabriel**
}

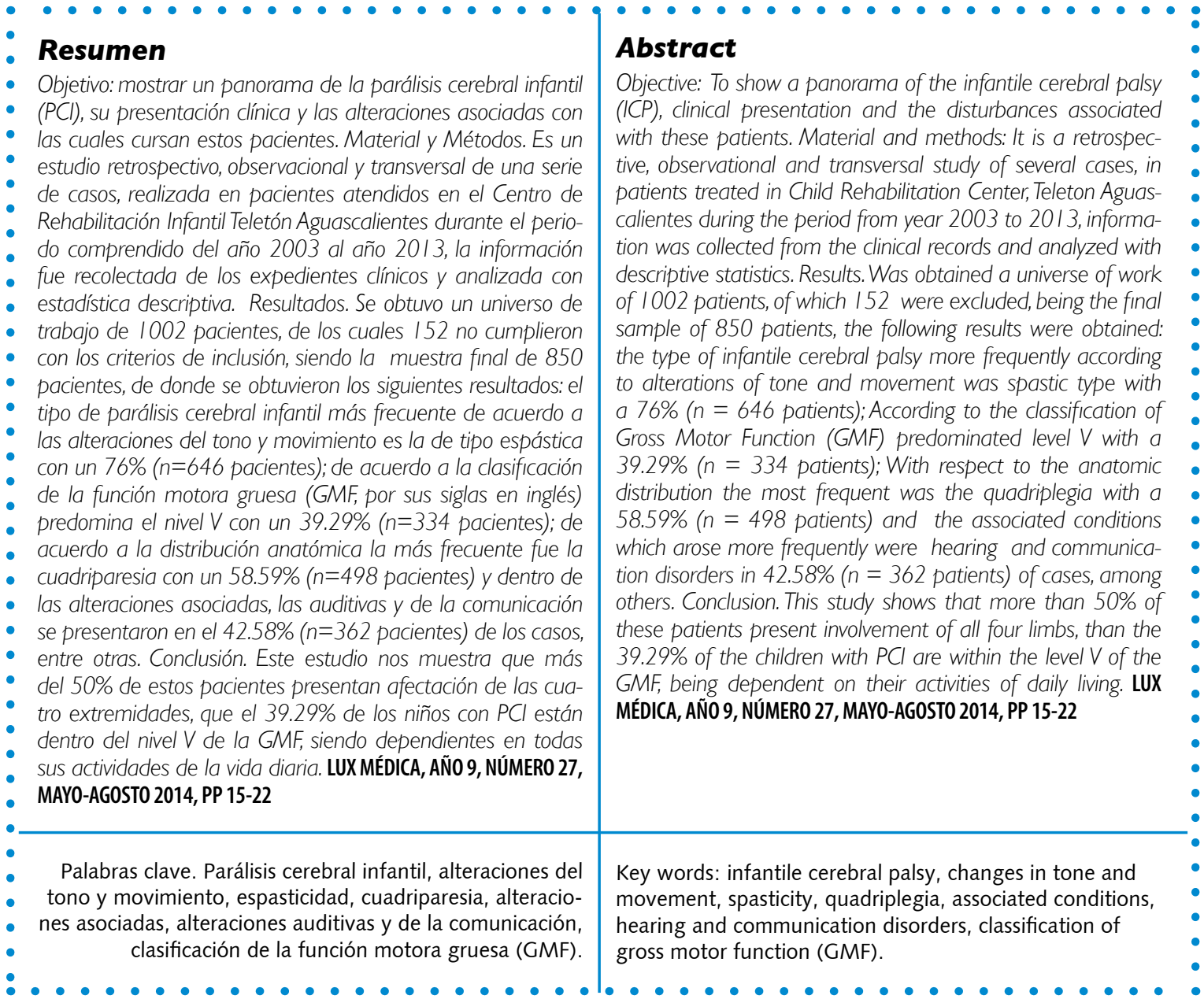

*Fisioterapeuta del Centro de Rehabilitación Infantil Teletón (CRIT) Aguascalientes. Licenciado en Terapia Física.

** Médico especialista en Medicina Física y Rehabilitación, subespecialidad en Rehabilitación Pediátrica. Médico acompañante del Centro de Rehabilitación Infantil Teletón Aguascalientes.

Fecha de recibido: 26 de mayo 2014

Fecha de aceptación:26 de junio 2014

Correspondencia: Aída del Valle Morales, Centro de Rehabilitación Infantil Teletón Aguascalientes. Avenida Heroico Colegio Militar \#600, Colonia Ferronales, CP 20059 Aguascalientes, Ags., México.Teléfonos 01 (44) 9910 2223, Fax: 01 (44) 9910 2223. Correo electrónico aidadelvalle80@yahoo.com.mx 


\section{Introducción}

Descrita por Little en 1840, la Parálisis Cerebral Infantil (PCI) engloba un grupo de trastornos del desarrollo del movimiento y la postura, que causan limitación en la actividad motriz. Se atribuye a un trastorno no progresivo que ocurre en un cerebro inmaduro y suele acompañarse de alteraciones sensoriales, cognitivas, de la comunicación, conducta o convulsivo. ${ }^{1-4}$ Tiene una prevalencia global de 2 a 3 por cada 1000 nacidos vivos. ${ }^{1,4}$ La presencia de factores de riesgo en la etapa prenatal, natal y postnatal así como la presencia de signos de alarma, permiten orientar el diagnóstico de la $\mathrm{PCl}$, ya que éste es fundamentalmente clínico; sin embargo, en casos de pacientes con antecedentes perinatales adversos, existen criterios basados en la neuroimagen que pueden ser predictivos de la enfermedad. ${ }^{5}$ La prematurez y el bajo peso al nacer son los factores de riesgo más importantes a nivel universal. ${ }^{6}$ Otros factores que ponen en riesgo el desarrollo adecuado del sistema nervioso central son: infección congénita o materna por virus de la rubéola, herpes simple, citomegalovirus, toxoplasmosis y sífilis (complejo TORCHS), pre-eclapmsia y eclampsia, enfermedades maternas graves, hipotermia o hipoglucemia del neonato, embarazo múltiple y parto distócico, entre otros. En casos donde se sospeche alguna enfermedad de fondo, debe realizarse el diagnóstico diferencial que permita determinar la verdadera causa de la alteración del desarrollo motriz y de ser necesaria su canalización. ${ }^{7}$

Existen diferentes formas de clasificar la $\mathrm{PCl}$, la más utilizada es la clasificación anatómica que se basa en el número de extremidades afectadas. ${ }^{5}$ Cuadriparesia es el término utilizado cuando se encuentran afectadas las cuatro extremidades, hemiparesia es cuando se encuentra afectado un hemicuerpo (extremidad superior e inferior del mismo lado), la paraparesia afecta solo a las extremidades inferiores, diparesia con afección de las cuatro extremidades con predominio de las inferiores y la triparesia que afecta a tres extremidades y se considera poco común. ${ }^{1,7}$

De acuerdo a las alteraciones del tono y movimiento se clasifica a la Parálisis Cerebral Infantil en espástica, discinética, atáxica, hipotónica y mixta. ${ }^{1-5}$ El término espástica se refiere a un incremento involuntario del tono muscular, es decir, existe contracción constante del músculo, provocando rigidez y acortamiento que interfiere con el movimiento y la función, ocurre sólo durante el estiramiento muscular y es causada por lesiones estructurales del cerebro, tallo cerebral, o la médula espinal. ${ }^{1-3,6}$ Póo Argüelles refiere que la parálisis cerebral de tipo discinética se caracteriza por una fluctuación y cambio brusco del tono muscular, presencia de movimientos involuntarios y persistencia de los reflejos arcaicos..$^{1-4,8} \mathrm{La}$ presentación de tipo atáxica se refiere a lesión en cerebelo, presentando alteraciones del equilibrio y coordinación ${ }^{1-5}$, la presentación mixta se presenta por una lesión de varias estructuras cerebrales, 
presentando combinaciones de diversos trastornos motores y extrapiramidales. ${ }^{1-7,9}$

El Sistema de Clasificación de la Función Motora Gruesa, es un sistema de evaluación viable, fiable, con significación pronóstica en pacientes con parálisis cerebral y los tratamientos que éstos reciben, se basa más en los logros funcionales de los niños que en sus limitaciones, enfatizando en el cumplimiento de las actividades diarias de la vida en la casa o en la comunidad. Se clasifica en cinco niveles con agrupaciones por edades, cada nivel representa el nivel más alto de movilidad que un niño puede llegar a conseguir entre los 6 y 12 años, el Nivel I Anda sin restricciones. Limitaciones para las habilidades motoras más avanzadas; es el más leve, los niños presentan alteraciones neuromotrices que no limitan su función, los pacientes en el Nivel I/ andan sin aparatos de ayuda para la movilidad: Limitaciones para andar fuera de casa y por la calle; no requieren de asistencia para moverse después de los 4 años, en el Nivel III Andan con ayuda de aparatos para la movilidad. Limitaciones para andar fuera de la casa y por la comunidad; requieren de asistencia para caminar permanente, en el Nivel IV Movilidad propia con limitaciones. Los niños son transportados o utilizan sus aparatos de movilidad fuera de casa y por la comunidad; solo logran sentarse con ayuda y en el Nivel $V$ Propia movilidad muy limitada incluso con sus ayudas tecnológicas; son totalmente dependientes en todas sus actividades de la vida diaria. ${ }^{9-13}$

Otra escala de valoración WeeFIM (Wee-Functional Independence Measure) - medición de la independencia funcional en niños - es un sistema para medir el desempeño funcional cotidiano en pacientes con discapacidad, consta de 18 elementos en los ámbitos de auto-atención, movilidad y cognición. Es aplicable a niños de 6 meses a 7 años así como a personas mayores que presenten retardos funcionales del desarrollo. ${ }^{14}$

Diversos trastornos pueden acompañar a la Parálisis Cerebral Infantil, dentro de los que se presentan con mayor frecuencia están las alteraciones de conducta y del aprendizaje ${ }^{15}$, alteraciones de la visión y del control de los movimientos oculares, epilepsia ${ }^{16,17}$, escoliosis $^{18}$, apoyo del pie o los pies en equino ${ }^{19}$, alteraciones de la deglución ${ }^{19,20}$, reflujo gastroesofágico ${ }^{21}$, desnutrición ${ }^{21,22}$, obesidad $^{22}$, luxación de cadera ${ }^{8}$, problemas auditivos y de la comunicación ${ }^{23}$.

La atención terapéutica de estos niños debe ser precoz para aprovechar al máximo la plasticidad cerebral y requiere un enfoque multidisciplinario, para conseguir el objetivo de un máximo desarrollo funcional y la mejor calidad de vida posible. ${ }^{5}$

El objetivo de este trabajo fue identificar un panorama de la $\mathrm{Pa}$ rálisis Cerebral Infantil y su presentación clínica, además de conocer las alteraciones asociadas que con mayor frecuencia se encuentran en pacientes que han sido atendidos en el CRIT Aguascalientes. 


\section{Material y métodos}

Es un estudio retrospectivo, observacional y transversal. Se revisaron las historias clínicas de los niños con diagnóstico de Parálisis Cerebral Infantil atendidos en el Centro de Rehabilitación Infantil Teletón Aguascalientes en el periodo del año 2003 al 2013 en las clínicas de lesión cerebral leve, moderada y severa que asisten en los turnos matutino y vespertino con edades de entre 4 a 18 años. Se incluyeron pacientes egresados, activos con evaluación WeeFIM terminada, recabando información sobre el tono muscular, su presentación topográfica, resultados del sistema de clasificación de la función motora gruesa y presentación de trastornos asociados; se excluyeron pacientes de nuevo ingreso, inactivos, en los que no se concluyó la valoración WeeFIM y pacientes dados de baja; se eliminaron los pacientes en los que no se encontraron datos suficientes. Posteriormente se creó una base de datos que se analizó con estadística descriptiva.

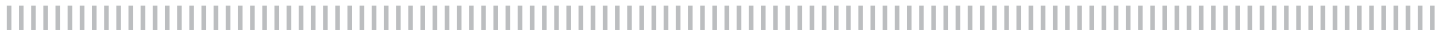

\section{Resultados}

De 1002 pacientes que han sido egresados o permanecen activos, 152 no cumplieron los criterios de inclusión quedando una muestra de 850 pacientes, en los cuales encontramos los siguientes resultados.

De acuerdo a las alteraciones del tono y movimiento, encontramos que los pacientes con $\mathrm{PCl}$ se distribuyeron de la siguiente manera: predominó la tipo espástica en un 76\% (646 pacientes), le siguió la tipo discinética en el 18.4\% (157 pacientes), la hipo- tónica en un 4.3\% (37 pacientes) y la atáxica en un 1.1\% (10 pacientes) (figura 1).

Dentro de la distribución anatómica la cual se basa en el total de miembros afectados, la población se distribuyó de la siguiente manera: cuadriparesia en el $58.59 \%$ de los casos (498 pacientes), hemiparesia en el $14.82 \%$ (126 pacientes), diparesia y paraparesia $15.52 \%$ (132 pacientes), triparesia $5.65 \%$ (48 pacientes) y doble hemiparesia en el $5.41 \%$ de los pacientes (46 pacientes) (figura 2).

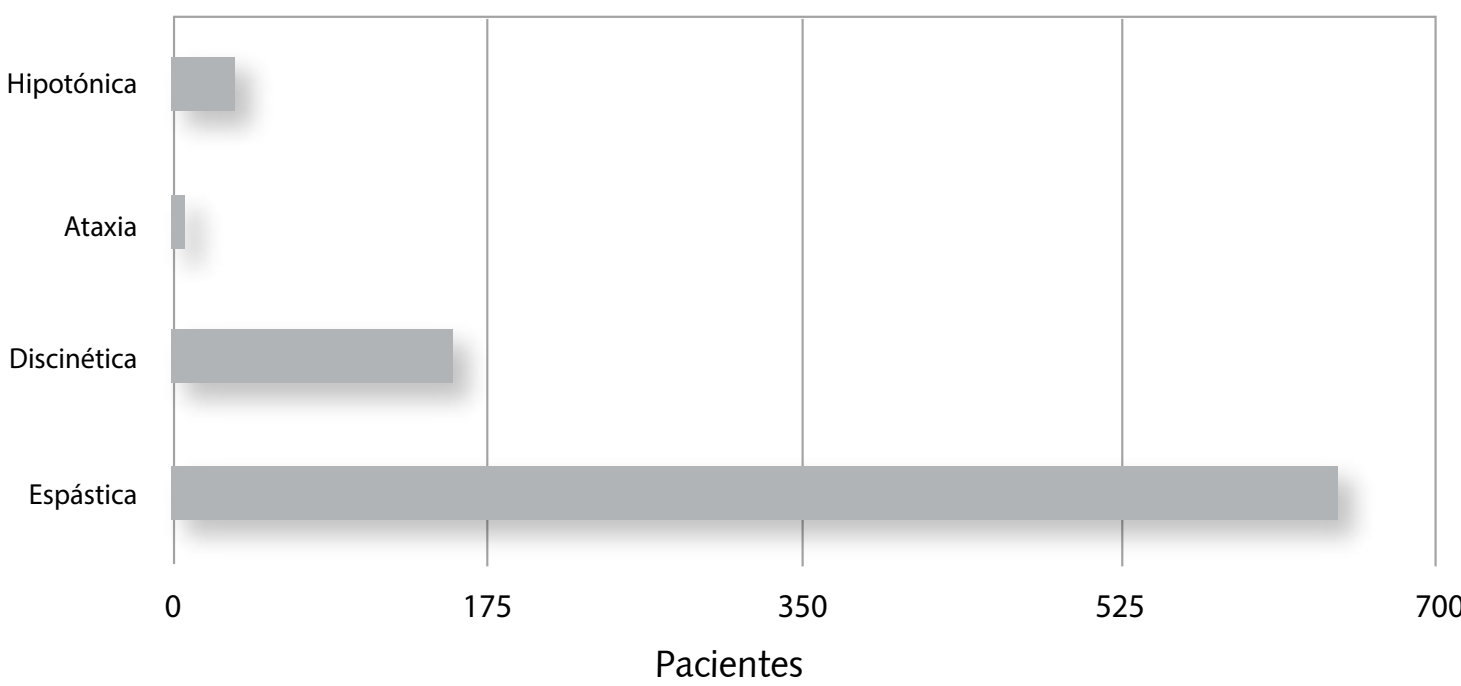

Figura 1. Distribución de la PCI de acuerdo a las alteraciones del tono y movimiento 


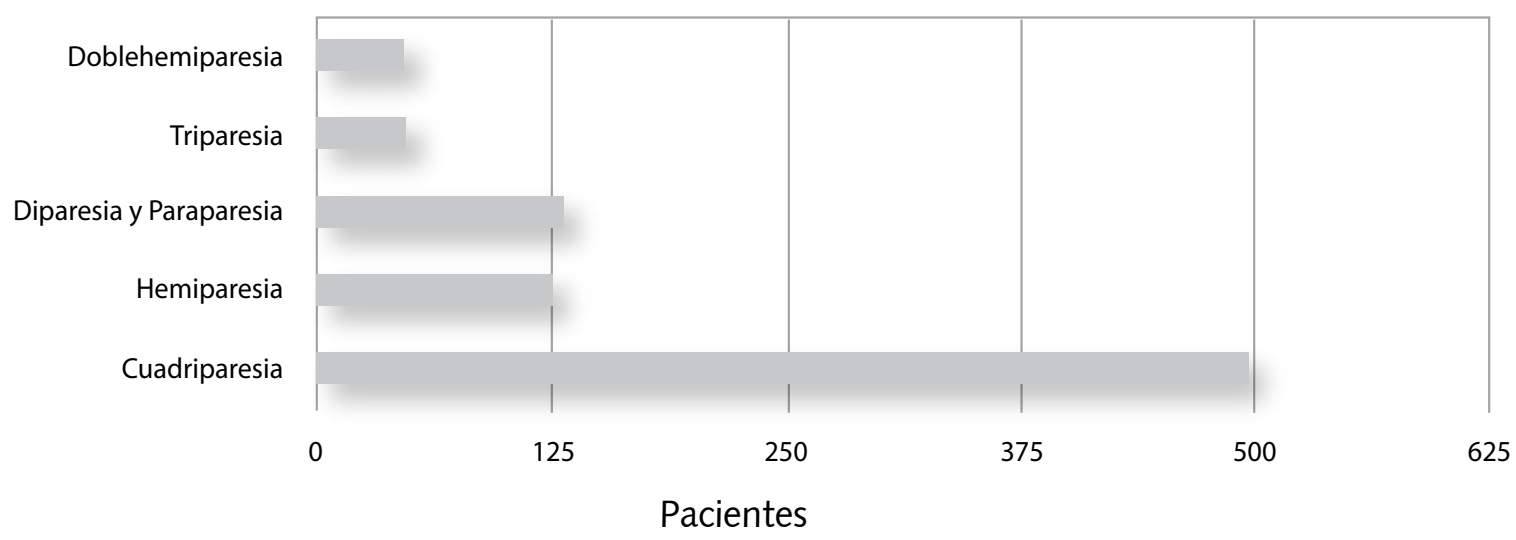

Figura 2. Distribución de los pacientes con PCI de acuerdo a su clasificación anatómica

De acuerdo al Sistema de la Clasificación de la Función Motora Gruesa de la parálisis cerebral infantil la población se distribuyó de la siguiente manera: al nivel I le correspondió el $8.12 \%$ (69 pacientes), al nivel II el $21.65 \%$ (184 pacientes), al nivel III el $18.47 \%$ (157 pacientes), al nivel IV el $12.47 \%$ (106 pacientes) y al nivel $\mathrm{V}$ el $39.29 \%$ (334 pacientes). (figura 3).

Encontramos además que los principales trastornos asociados a la $\mathrm{PCl}$ fueron en orden decreciente: las alteraciones auditivas y de la comunicación 42.58\% (362 pacientes), epilepsia $22.58 \%$ (192 pacientes), alteraciones visuales $15.05 \%$ (128 pacientes), alteraciones de la conducta y del aprendizaje $14.82 \%$ (126 pacientes), luxación o subluxación de la cadera $12.70 \%$ (108 pacientes), desnutrición 11.41\% (97 pacientes), enfermedad por reflujo gastroesofágico 3.64\% (31 pacientes), escoliosis $3.41 \%$ (29 pacientes), obesidad $1.99 \%$ (17 pacientes), enfermedades pulmonares $1.52 \%$ (13 pacientes), alteración de la deglución $1.17 \%$ (10 pacientes) y pie equino $0.82 \%$ (7 pacientes). (figura 4 ).

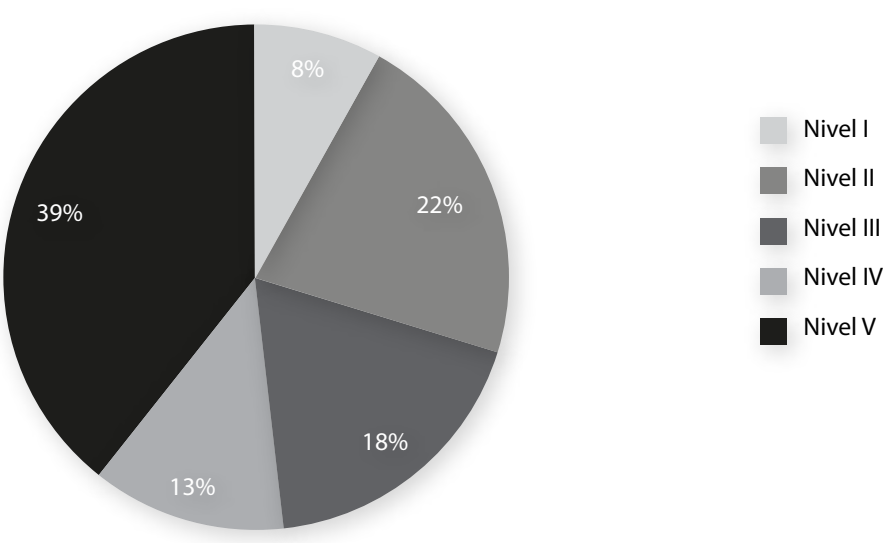

Figura 3. Distribución de los pacientes con PCI de acuerdo a la clasificación de la Función Motora Gruesa. 


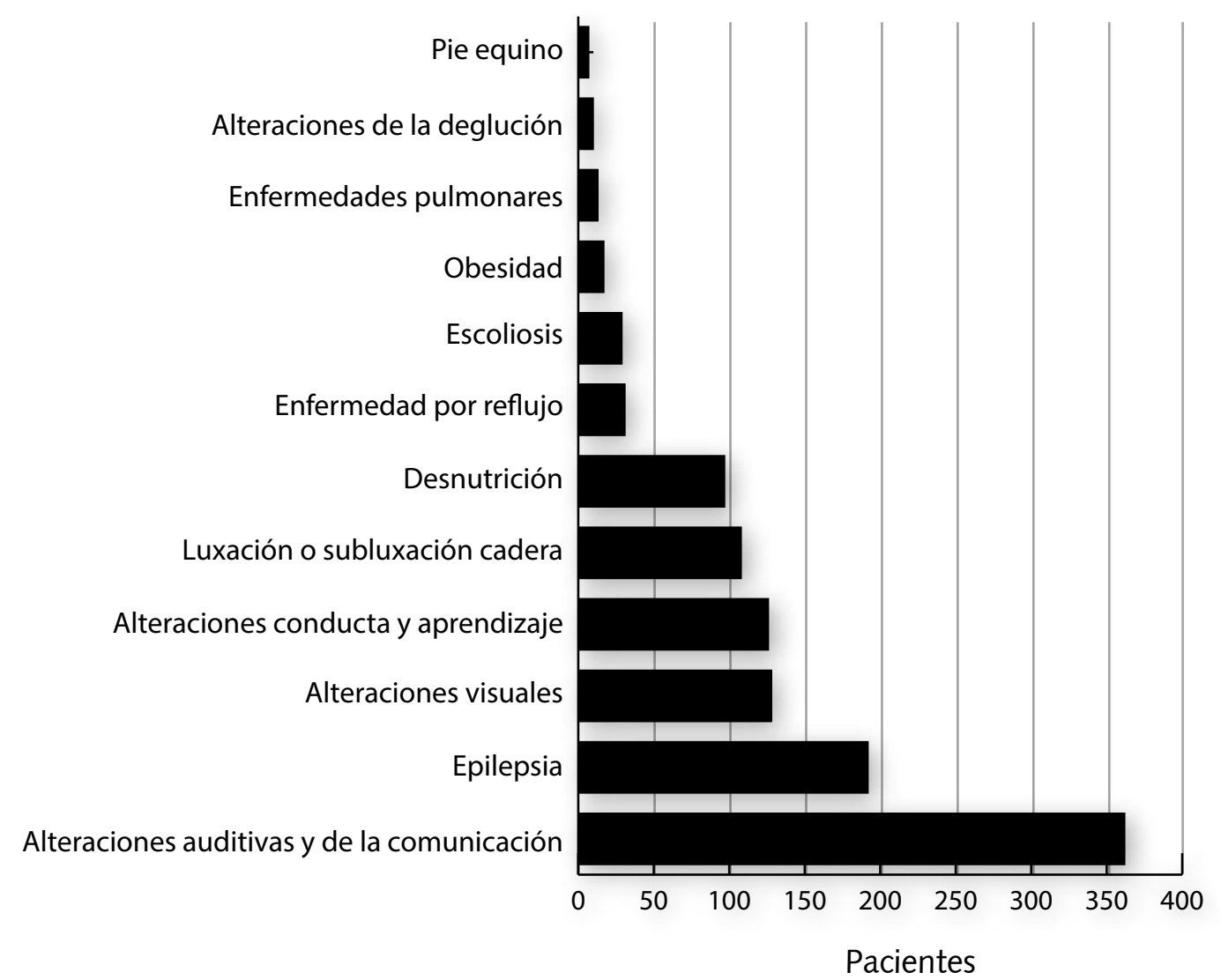

Figura 4. Distribución de los trastornos asociados en los pacientes con PCI

\section{Discusión}

Sabemos que la $\mathrm{PCl}$ representa un problema de salud pública ya que la discapacidad afecta al individuo y sus familias en las esferas biopsicosocial y espiritual, pues genera en el paciente alteraciones del movimiento afectando la independencia en sus actividades básicas de la vida diaria, su comunicación, autoestima y la vinculación con el entorno, alterándose así la dinámica de la familia.

En el 2008, Pérez y Hernández ${ }^{12}$ reportaron que el $58.60 \%$ de los casos presentaban cuadriparesia, resultados que son similares a nuestro estudio $(58.59 \%)$, a diferencia de Pérez Flores ${ }^{7}$ y colaboradores quienes publicaron en un estudio realizado en el 2012 un $51.4 \%$, porcentaje más bajo que nuestros resultados pero aun conservando cierta similitud, no así con los resultados publicados por el departamento de pediatría del Hospital Infantil de Bangalore en la India donde Chitra ${ }^{1}$ tiene reportado que en el $10-15 \%$ de sus pacientes con $\mathrm{PCl}$ predomina la cuadriparesia, lo que nos hace cuestionar si estas diferencias se deben a los niveles de atención en sector salud.

En lo referente a la Clasificación de la Función Motora Gruesa el Nivel $\mathrm{V}$ es el más frecuente en nuestra investigación, con un $39.29 \%$, resultados que coinciden en los expuestos por Piana ${ }^{13}$ en 2010 en el artículo "Evaluación neuromotora de 
pacientes con parálisis cerebral espástica tratados con cirugía ortopédica en el Instituto Nacional de Rehabilitación, en la Ciudad de México" reportando un 50\% de pacientes con nivel $\mathrm{V}$ en la Clasificación de la Función Motora Gruesa, lo cual significa que tenemos una amplia población de niños que dependen de asistencia y cuidados en todas sus actividades de la vida diaria. Por otro lado, Westbom ${ }^{10}$ en un estudio realizado en un paciente con $\mathrm{PCl}$ de 4 a 11 años de edad en Suecia, reportó predominio del nivel I que corresponde a la afectación motriz más leve, lo cual nos lleva a ratificar las similitudes que existen en los centros especializados, en donde sus servicios se dedican a la atención de personas con discapacidad neuromusculoesquelética, centrando su atención en evaluar la funcionalidad del paciente y la preocupación de poder incluirlo en la sociedad.

Sobre las alteraciones del tono y movimiento, en un estudio epidemiológico realizado en España en el 2007 por Robaina ${ }^{11}$ y colaboradores reportaron que el $70-80 \%$ de la población estudiada presentaban alteraciones de tipo espástica, resultados similares a los encontrados es este estudio donde la alteración tipo espástica la encontramos en el $76 \%$ de los casos.

Respecto a los trastornos asociados en los pacientes con $\mathrm{PCl}$ estudiados, las alteraciones auditivas y de la comunicación fueron las más frecuentes $(42.58 \%)$, y no encontramos reportes coincidentes, sólo Chitra $^{1}$ y Poó $^{23}$ hacen referencia de estos trastornos en menor escala, por lo cual se recomienda profundizar más en próximos trabajos sobre este trastorno.

$\mathrm{La} \mathrm{PCl}$ es un trastorno que limita la adquisición de posturas y movimientos normales por lo tanto es la causa más frecuente de discapacidad motora en la edad pediátrica, trascendiendo a lo largo de la vida; tiene implicaciones médicas, sociales y educacionales. Por esto y ante la poca información a nivel nacional es necesario contar con una estadística real que nos permita conocer la situación actual para así planificar de forma adecuada la asistencia a estos pacientes y crear evaluaciones motrices que se ajusten a la antropometría de la población mexicana y actuar en todos los niveles de la salud. Además de esto es indispensable un intenso programa de prevención de la discapacidad con estrategias para mejorar la atención materno-infantil, así como la derivación oportuna de pacientes con factores de riesgo o signos de alarma neurológica a programas de estimulación múltiple. Para todos estos pacientes es necesario una atención multidisciplinaria, así como la creación de redes de apoyo entre padres de familia quienes necesitan capacitación para atender a sus hijos.

\section{Conclusiones}

Los resultados encontrados en esta investigación nos muestran que más del $50 \%$ de estos pacientes tiene afectadas las cuatro extremidades, casi el $40 \%$ de los niños con parálisis cerebral infantil presenta un nivel $V$ dentro de la Clasificación de la Función Motora Gruesa, lo que traduce que estos pacientes son dependientes en todas sus actividades de la vida diaria y además tienen en su mayoría trastornos asociados a nivel sensorial, intelectual y deformidades, limitando aún más su funcionalidad. Es apremiante entonces, contribuir al mejoramiento de la salud de aquellas personas que nacieron o adquirieron determinadas enfermedades y brindarles mayores expectativas en su calidad de vida. 


\section{Bibliografía}

1. Chitra S. Nandini M. Cerebral palsy-definition, classification, etiology and early diagnosis. Indian Journal of Pediatrics.2005;72:865-868.

2. Krigger K. Cerebral palsy:an overview. American Family Physician. 2006;73:91-99.

3. Robaina M. Riesgo S.Definición y clasificación de la parálisis cerebral. Revista de Neurología.2007; 45:110-117.

4. Goldstein M. Rosenbaum P. Paneth N.Proposed definition and classification of cerebral palsy, April 2005.Developmental Medicine y Child Neurology. 2005; 47 : 571-576.

5. Lorente I. La parálisis cerebral. Actualización del concepto, diagnóstico y tratamiento. Pediatría Integral.2007; XI(8):687-698.

6. Avedis Aznavurian A. Aguilar Rebolledo. Espasticidad ¿Qué es, qué no es?. Plasticidad y Restauración Neurológica. 2005; 5(2): 152-159.

7. Pérez F. Macías H. Guerra N. Cedillo M. Parodi C. Evaluación de la función motora gruesa y habilidad manual como parámetros de integración escolar de adolescentes con parálisis cerebral. Presentación cartel en III Congreso Internacional de investigación en Rehabilitación.

8. Hägglund $G$, Lauge $H$, Wagner $P$. Characteristics of children with hip displacement in cerebral palsy. Bio Med Central Musculoskeletal Dissorders.2007;8:101-107.

9. Bialik G. Givon U.Cerebral palsy: classification and etiology. Acta Orthopaedica et Traumatologica.2009;43(2):77-80.

10. Westbom L. Hagglund G. Nordmark E.Cerebral palsy in a total population of $4-11$ year olds in southern Sweden. Prevalence and distribution according to different CP classification systems. Bio Med Central Pediatrics.2007;7:41-49.

11. Robaina G. Riesgo S. Robaina M. Evaluación diagnóstica del niño con parálisis cerebral. Revista Cubana de Pediatría. Disponible en scielo.sld.cu/scielo. php?script=sci_arttext\&pid...75312007000200007.

12. Pérez L. Hernández A. Parálisis cerebral infantil: características clínicas y factores relacionados con su atención. Revista Archivo Médico de Camagüe y versión ISSN 1025. 2008.

13. Piana A. Viñals C. Del Valle M. Redón T. Peralta S. León L. Evaluación neuromotora de pacientes con parálisis cerebral espástica tratados con cirugía ortopédica en el Instituto Nacional de Rehabilitación. Acta Ortopédica Mexicana 2010; 24 (5):331-337.

14. Segovia López Saúl, Ortiz Ortega Enrique. Evaluación del desempeño funcional de pacientes con parálisis cerebral severa con el instrumento WeeFIM en el CRIT Estado de México. Revista Mexicana de Medicina Fisica y Rehabilitación, 2005(17):54 -59.

15. Bringas A. Fernández A. García C. Barrera M. Parálisis cerebral infantil: estudio de 250 casos. Revista de Neurología. 2002;35(9):812-817.

16. Malangón J. Parálisis cerebral. Medicina (Buenos Aires) 2007; 67 (6/1): 586-592.

17. Morrell D. Pearson M. Sauser D. Progressive bone and joint abnormalities of the apine and lower extremities in cerebral palsy. RadioGraphics.2002;22:257-268.

18. Wren T. Rethlefsen S. Kay R. Prevalence of specific gait abnormalities in children with cerebral palsy. Journal Pediatrics Orthopedic.2005;25:79-83.

19. Benfer K. Weir K. Bell K. Ware R. Longitudinal cohort protocol study of oropharyngeal dysphagia: relationships to gross motor attainment, growth and nutritional status in preschool children with cerebral pals. Bio Medical Journal. 2012;0:e001460.

20. González D. Díaz J. Bousoño C. Patología gastrointestinal en niños con parálisis cerebral infantil y otras discapacidades neurológicas. Anales de Pediatría.2010;73(6):361.e1-361.e6.

21. Sanchez, V.Weight - Based nutritional diagnosis of Mexican children and adolescent with neuromotor disabilities. Bio Med Central Research Notes 2012;5:218-225.

22. Del Águila A. Áibar P. Características nutricionales de niños con parálisis cerebral. Anales de la Facultad de Medicina Universidad Nacional Mayor de San Marcos. 2004.

23. Póo P. Parálisis cerebral infantil. Asociación Española de Pediatría. 2008: 271-279. 